Larissa de Araúijo Correia Teixeira' ${ }^{1}$

Ohttps://orcid.org/0000-0003-0735-8016

Ricardo Alves Costa

Ohtps://orid.org/0000-0001-9438-038X

Roberta Machado Pimentel Rebello

de Mattos'

Ohttps://orcid.org/0000-0002-7275-2522

Déborah Pimentel²

Ohttps://orcid.org/0000-0003-2102-7125

\section{Saúde mental dos estudantes de Medicina do Brasil durante a pandemia da coronavirus disease 2019}

\author{
Brazilian medical students' mental health during \\ coronavirus disease 2019 pandemic
}

DOl: $10.1590 / 0047-2085000000315$

\section{RESUMO}

Objetivo: $\bigcirc$ objetivo desta pesquisa é verificar a prevalência de sintomas de sofrimento psíquico em estudantes do curso de Medicina durante a pandemia da COVID-19. Métodos: Trata-se de um estudo transversal e exploratório que avaliou 656 estudantes do curso de Medicina do Brasil. Os dados foram coletados, em maio e junho de 2020, por meio de dois instrumentos autoaplicáveis. O primeiro foi um questionário elaborado pelos próprios autores para avaliar o perfil social, demográfico e cultural da população. Para o rastreamento de indícios de sofrimento psíquico, utilizou-se o Self-Report Questionnaire, um questionário com 20 itens divididos em quatro domínios. Durante a análise de dados, as associações entre variáveis categóricas foram testadas por meio do teste qui-quadrado de Pearson. O nível de significância adotado foi de 5\%. Resultados: A prevalência de indivíduos com indícios de sofrimento psíquico foi de $62,8 \%$. São fatores de risco para o adoecimento mental durante a pandemia da COVID-19: ser do sexo feminino, estar nos dois primeiros anos do curso, relatar má adaptação ao ensino a distância, apresentar dificuldade de concentração, preocupar-se com o atraso da graduação, ter um diagnóstico prévio de transtorno mental, morar com alguém que precisa trabalhar fora de casa, ser incapaz de manter hábitos saudáveis e ter medo de ser infectado pelo vírus. Conclusão: Este estudo demonstrou que os indícios de sofrimento psíquico estão elevados entre estudantes de Medicina durante a pandemia da COVID-19. Além disso, também foi possível concluir que há fatores protetores para o adoecimento mental.

\section{PALAVRAS-CHAVE}

Saúde mental, COVID-19, isolamento social, estresse psicológico, estudantes de Medicina.

\section{ABSTRACT}

Objective: The objective of the research is to verify the prevalence of psychological distress symptoms in medical students during the COVID-19 pandemic. Methods: This is a cross-sectional and exploratory study that evaluated 656 medical students in Brazil. Data were collected between May and June 2020, through two self-administered instruments. The first was a questionnaire prepared by the authors themselves to assess the social, demographic and cultural profile of the population. For the screening of signs of psychological distress, the Self-Report Questionnaire was used, a questionnaire with 20 items divided into four domains. During data analysis, associations between categorical variables were tested using Pearson's chi-square test. The significance level adopted was 5\%. Results: The results show that the prevalence of individuals with signs of psychological distress was $62.8 \%$. Risk factors for mental illness during the COVID-19 pandemic are being female, being in the first two years of the course, reporting poor adaptation to Distance Learning, having difficulty concentrating, worrying about the delay of graduation, have a previous diagnosis of mental disorder, live with someone who needs to work outside the home, inability to maintain healthy habits and fear being infected by the virus. Conclusion: We conclude that the signs of psychological distress are high among medical students during the COVID-19 pandemic. In addition, it was also possible to conclude that there are protective factors for mental illness.

KEYWORDS

Mental health, COVID-19, social isolation, psychological stress, medical students. 


\section{INTRODUÇÃO}

A COVID-19 (do inglês: coronavirus disease 2019) consiste em uma doença contagiosa decorrente da infecção pelo vírus SARS-CoV-2 da família Coronavírus; o primeiro caso registrado da doença foi em dezembro de 2019 em Wuhan, na China'. Uma peculiaridade dessa doença é que a capacidade de transmissão do vírus é muito alta ${ }^{2}$ e a doença pode evoluir com sérias complicações², podendo o paciente necessitar de internação em leito de terapia intensiva.

Dessa forma, o enfrentamento da atual pandemia baseia-se na adoção de medidas de distanciamento social, por meio do isolamento social domiciliar e evitando aglomerações ${ }^{3}$. Diante disso, grande parte do país encontra-se num estado de redução de convívio social, com escolas e universidades paralisadas e diversos pontos de encontro (igrejas, bares, praças, praias etc.) interditados $s^{4,5}$.

Na literatura está bem documentado que uma rede de apoio psicossocial constituída de vínculos e relações sociais é fundamental no contexto da atenção em saúde mental ${ }^{6}$. Assim, o suporte social é considerado como um fator capaz de proteger e promover a saúde mental. A literatura demonstra que há características específicas relacionadas com o desenvolvimento de sintomas mentais, tais como redes compostas por um número reduzido de pessoas e baixo índice de utilização dos recursos disponíveis para integração social77.8.

É válido ressaltar que os estudantes universitários, em particular os do curso de Medicina, compõem um grupo vulnerável para o adoecimento mental. A alta prevalência de transtornos mentais é decorrente de vários fatores, como a exposição a uma carga horária extenuante, ao estresse crôni$\mathrm{CO}$, às cobranças pessoais e externas, e à hostilidade de docentes e, até mesmo, de discentes, que parecem contribuir com a vulnerabilidade dos alunos de tal forma que possa haver o desenvolvimento de doenças mentais ${ }^{9-11}$.

Diante disso, o presente estudo tem o objetivo de verificar a prevalência de sintomas de sofrimento psíquico em estudantes do curso de Medicina do Brasil no contexto das medidas de distanciamento social impostas na pandemia da COVID-19 no ano de 2020.

\section{MÉTODOS}

\section{População e amostra}

Trata-se de um estudo transversal, exploratório e quantitativo que avaliou uma população de 656 estudantes do curso de Medicina de diversas universidades de todas as regiões do Brasil ${ }^{12}$. Os critérios de inclusão para este estudo foram: estudantes de Medicina, maiores de 18 anos, que estejam realizando o curso no Brasil e que aderiram às medidas de isolamento social durante a pandemia da COVID-19.

O recrutamento dos participantes foi realizado por meio do método bola de neve, isto é, os pesquisadores convidaram estudantes membros de associações e agremiações estudantis das escolas médicas do país e solicitaram que eles convidassem novos participantes, e assim por diante.

Atualmente, no Brasil, há 305 cursos de Medicina. Em 2018, cerca de 25 mil médicos se formaram no país. Logo, estima-se que, multiplicando pelo número de anos cursados na graduação de Medicina, há 150 mil estudantes cursando no país aproximadamente (Conselho Federal de Medicina - CFM, 2018). Considerando o pior cenário estatístico que maximiza o erro na estimação da quantidade de estudantes de Medicina do Brasil que adquiriram os sintomas de sofrimento psíquico, seria de 50\%. Portanto, supondo o número de 150 mil estudantes, com uma margem de erro de 5\%, uma confiança de 95\%, são necessários pelo menos 384 participantes $^{13}$.

\section{Coleta de dados}

A coleta de dados foi realizada no período de maio a junho de 2020 por meio da plataforma de administração de pesquisas on-line do Google. A escolha dessa ferramenta foi motivada pela vontade dos pesquisadores de garantir a proteção dos respondentes, tendo em vista as medidas de contingenciamento domiciliar do governo durante o enfrentamento da pandemia. Antes de responder aos questionários da pesquisa, os participantes tiveram acesso aos meios de contato dos pesquisadores e às informações sobre os objetivos da pesquisa na íntegra. Por meio da plataforma virtual, os participantes puderam concordar eletronicamente com o Termo de Consentimento Livre e Esclarecido e com a participação no estudo. Este trabalho foi aprovado pelo Comitê de Ética e Pesquisa da Universidade Federal de Sergipe, Aracaju, Sergipe, com o CAAE de número: 31021520.0.0000.5546.

\section{Questionários}

Durante a coleta de dados, os participantes responderam a dois instrumentos autoaplicáveis. O primeiro foi um questionário elaborado pelos próprios autores para avaliar o perfil social, demográfico e cultural da população estudada. A ferramenta em questão possui 21 itens, divididos em quatro grandes grupos: cinco itens referentes ao perfil sociodemográfico, cinco itens relacionados às atividades acadêmicas durante a pandemia, três itens ligados à saúde mental (que inclui a informação de diagnóstico de transtorno mental prévio realizado por um profissional graduado em Medicina) e, por fim, oito itens referentes aos aspectos sociais e hábitos de vida durante a pandemia. Todas as questões utilizadas nesse instrumento estão demonstradas nas Tabelas 1, 2, 3 e 4, dispostas conforme a divisão supracitada.

Para o rastreamento de indícios de sofrimento psíquico, foi utilizado o Self-Report Questionnaire (SRQ-20), um questionário autoaplicável com respostas tipo sim e não composto por 20 itens agrupados em quatro domínios: humor, 
vitalidade, sintomas somáticos e pensamentos depressiVOS $^{14,15}$. O SRQ-20 é um instrumento criado por Hardig em 1980 e validado no Brasil por Williams em 1986 16,17. Esse teste favorece uma triagem de adoecimento psíquico, sugerindo suspeita, sem, no entanto, atribuir diagnóstico específico. Nesse questionário, tomam-se sete ou mais respostas positivas como o ponto de corte para definir se o indivíduo apresenta indícios de sofrimento psíquico ou não, isto é, o escore de corte do SRQ-20 para este estudo foi definido em 7: SQR $>7$ se refere aos indivíduos que resultaram não ter indícios de sofrimento psíquico e $\mathrm{SQR}<7$ se refere aos indivíduos que resultaram ter indícios de sofrimento psíquico a partir do questionário ${ }^{18,19}$.

\section{Análise de dados}

As variáveis categóricas foram descritas por meio de frequências percentuais relativas e absolutas. As associações entre variáveis categóricas foram testadas por meio do teste qui-quadrado de Pearson. O nível de significância adotado foi de 5\%. O software utilizado foi o R Core Team 2020.

\section{RESULTADOS}

\section{Características sociodemográficas}

Em nossa amostra de 656 universitários, percebe-se que há um predomínio de acadêmicos do gênero feminino $(80,6 \%)$ em relação aos acadêmicos do gênero masculino (19,4\%).

Quanto ao estado civil, 93,6\% são solteiros, embora 40,1\% do total de estudantes estejam em um relacionamento; $5,6 \%$ são casados e 0,8\%, separados ou divorciados.

A maioria dos respondentes se enquadra na faixa etária entre os 18 e 23 anos (70,7\%), 22,3\% se encontram entre 24 e 28 anos e apenas 7\% têm 29 anos ou mais. Já quanto à religião, os maiores destaques foram católicos $(65,9 \%)$ e sem práticas religiosas (22,7\%). Quanto à região de residência, $67,4 \%$ são do Nordeste, 17,2\% do Sudeste, 10,5\% do Sul, 2,6\% do Centro-Oeste e 2,3\% do Norte.

Estudantes de todos os anos do curso de Medicina responderam aos questionários. Do total de estudantes, 19,8\% e $27,1 \%$ encontravam-se no primeiro e no segundo ano do curso, respectivamente. Ademais, 25,8\% cursavam o terceiro ano e $8,5 \%$, o quarto ano. Por fim, $12,5 \%$ e $6,3 \%$ encontravam-se no quinto e no sexto ano do curso, respectivamente.

\section{Indícios de sofrimento psíquico}

De acordo com o SRQ-20, a prevalência de indivíduos com indícios de sofrimento mental foi de $62,8 \%$. A Tabela 1 correlaciona a presença de adoecimento psíquico com as características sociodemográficas da população estudada.

Os sintomas somáticos mais frequentes são: sono comprometido $(54,6 \%)$, desconforto estomacal $(51,7 \%)$ e cefaleia (49,2\%). Além disso, os estudantes relataram má digestão (36,4\%), falta de apetite (27\%) e tremores nas mãos (23,3\%).
Com relação à vitalidade, grande parte dos estudantes sentem-se com dificuldade para realizar as atividades com satisfação e sentem-se cansados facilmente $(71,6 \%$ e $64,9 \%$, respectivamente). Ademais, metade dos participantes relatou dificuldade para tomar decisões e para pensar com clareza (56,7\% e 53,2\%, respectivamente). Por fim, 52,7\% sentem-se cansados o tempo todo e 31,6\% referem ter dificuldade nos trabalhos.

Quanto ao humor, 83,8\% dos estudantes estão tensos, nervosos ou preocupados, 70,3\% sentem-se tristes, 50,5\% assustam-se com facilidade e $41,3 \%$ têm chorado mais do que de costume.

Em relação aos pensamentos depressivos, 55\% afirmam estar perdendo o interesse pelas coisas e $39,2 \%$ sentem-se inúteis ou sem préstimo. Além disso, 18,6\% relataram o sentimento de incapacidade sobre desempenhar um papel útil em sua vida. É válido mencionar que 9,1\% apresentam ideação suicida.

Tabela 1. Perfil sociodemográfico segundo a presença ou não de sofrimento psíquico

\begin{tabular}{|c|c|c|c|}
\hline & \multicolumn{2}{|c|}{ SRQ-20 } & \multirow[b]{2}{*}{ Valor-p } \\
\hline & $\begin{array}{c}>7 \\
n(\%)\end{array}$ & $\begin{array}{c}\leq 7 \\
\mathrm{n}(\%)\end{array}$ & \\
\hline \multicolumn{4}{|l|}{ Idade } \\
\hline $18-23$ & $305(74)$ & $159(65,2)$ & 0,036 \\
\hline $24-28$ & $84(20,4)$ & $62(25,4)$ & \\
\hline 29 ou mais & $23(5,6)$ & $23(9,4)$ & \\
\hline \multicolumn{4}{|l|}{ Sexo biológico } \\
\hline Sexo feminino & $359(87,1)$ & $170(69,7)$ & $<0,001$ \\
\hline Sexo masculino & $53(12,9)$ & $74(30,3)$ & \\
\hline \multicolumn{4}{|l|}{ Estado civil } \\
\hline Solteiro e sozinho & $231(56,1)$ & $120(49,2)$ & 0,074 \\
\hline Solteiro, mas namorando & $160(38,8)$ & $103(42,2)$ & \\
\hline $\begin{array}{l}\text { Casado, união estável ou vive } \\
\text { maritalmente }\end{array}$ & $20(4,9)$ & $17(7)$ & \\
\hline Separado ou divorciado & $1(0,2)$ & $4(1,6)$ & \\
\hline \multicolumn{4}{|c|}{ Ano da graduação em que se encontra } \\
\hline $1^{\circ}$ ano & $85(20,6)$ & $45(18,4)$ & 0,001 \\
\hline $2^{\circ}$ ano & $119(28,9)$ & $59(24,2)$ & \\
\hline $3^{\circ}$ ano & $104(25,2)$ & $65(26,6)$ & \\
\hline $4^{\circ}$ ano & $45(10,9)$ & $11(4,5)$ & \\
\hline $5^{\circ}$ ano & $40(9,7)$ & $42(17,2)$ & \\
\hline $6^{\circ}$ ano & $19(4,6)$ & $22(9)$ & \\
\hline \multicolumn{4}{|l|}{ Religião } \\
\hline Cristianismo & $263(63,8)$ & $169(69,3)$ & 0,373 \\
\hline Budismo & $2(0,5)$ & $2(0,8)$ & \\
\hline Umbanda & $5(1,2)$ & $3(1,2)$ & \\
\hline Outro & $38(9,2)$ & $25(10,2)$ & \\
\hline Nenhum & $104(25,2)$ & $45(18,4)$ & \\
\hline
\end{tabular}




\section{Atividades acadêmicas e a pandemia da COVID-19}

Entre os estudantes de Medicina, 71,8\% tiveram suas atividades acadêmicas continuadas por meio de plataformas virtuais, ao passo que $26,2 \%$ tiveram suas obrigações interrompidas e $2 \%$, continuadas de forma presencial.

Dos respondentes, 79,4\% dos alunos apontaram má adaptação ao ensino à distância (EAD) e, desse total, 78,5\% acreditam que não estão aprendendo o conteúdo adequadamente. Em nosso grupo, 80,2\% relataram dificuldade para estudar após a instalação das medidas de isolamento social por causa de redução da concentração ou de dificuldade de adaptação às plataformas virtuais. Ademais, $72,3 \%$ se preocupam com a perda ou o atraso do semestre e $69,8 \%$ se preocupam com o acúmulo de assuntos quando houver o retorno das aulas presenciais.

A Tabela 2 demonstra a relação entre aspectos relacionados às atividades acadêmicas durante a pandemia da COVID-19 e a presença de indícios de sofrimento psíquico de acordo com o SRQ-20. A má adaptação ao EAD, a dificuldade de concentração e a preocupação com o acúmulo de assuntos para o retorno presencial e com a perda ou atraso do semestre demonstraram relação com o adoecimento mental $(p<0,001)$.

\section{Saúde mental dos estudantes de Medicina}

Entre os participantes da nossa amostra, 28,8\% possuem um diagnóstico prévio de transtorno mental realizado por um profissional de saúde graduado em Medicina, resultando na seguinte distribuição: 19,7\% com transtornos de ansiedades, $11,7 \%$ com transtornos depressivos, 2,4\% com transtorno de déficit de atenção e de hiperatividade (TDAH), 1,7\% com transtorno obsessivo-compulsivo (TOC), 1,5\% com transtorno da personalidade borderline, 1,1\% com transtorno bipolar e $0,8 \%$ com outros transtornos.

Ademais, 81,4\% dos participantes relatam ter percebido em si alguma mudança psicológica ou comportamental durante o período de isolamento. Todavia, apenas 22,1\% expressaram o desejo de retorno dos pesquisadores para um possível contato a fim de obter acompanhamento psicoterápico em um espaço disponibilizado por eles.

A Tabela 3 demonstra a relação entre a saúde mental da população estudada e a presença de indícios de sofrimento psíquico de acordo com o SRQ-20. Percebe-se uma associação entre indivíduos com diagnóstico prévio de transtorno mental e o adoecimento mental durante a pandemia $(p<0,001)$. Além disso, há correlação entre a autopercepção de mudança psicológica ou comportamental e o sofrimento psíquico $(p<0,001)$.

Tabela 2. Relação entre aspectos relacionados às atividades acadêmicas durante a pandemia da COVID-19 e a presença de sofrimento psíquico

\begin{tabular}{|c|c|c|c|}
\hline & \multicolumn{2}{|c|}{ SRQ-20 } & \multirow[b]{2}{*}{ Valor-p } \\
\hline & $\begin{array}{c}>7 \\
\mathrm{n}(\%)\end{array}$ & $\begin{array}{c}\leq 7 \\
\mathrm{n}(\%)\end{array}$ & \\
\hline \multicolumn{4}{|l|}{ Durante a pandemia da COVID-19, suas atividades acadêmicas foram: } \\
\hline Totalmente interrompidas & $108(26,2)$ & $64(26,2)$ & 0,452 \\
\hline Continuadas por meio de EAD & $298(72,3)$ & $173(70,9)$ & \\
\hline Continuadas de forma presencial & $6(1,5)$ & $7(2,9)$ & \\
\hline \multicolumn{4}{|l|}{ Você está se adaptando bem ao ensino a distância (EAD)? } \\
\hline Sim, tenho uma boa conexão com o EAD. & $76(18,4)$ & $101(41,4)$ & $<0,001$ \\
\hline Não, tenho dificuldade para lidar com as plataformas. & $47(11,4)$ & $23(9,4)$ & \\
\hline Não, acho que não estou aprendendo. & $191(46,4)$ & $66(27)$ & \\
\hline A faculdade não aderiu ao EAD. & $98(23,8)$ & $54(22,1)$ & \\
\hline \multicolumn{4}{|c|}{ Você está preocupado com a reposição futura pela faculdade das práticas não abordadas pelo EAD? } \\
\hline Sim, me preocupo com o acúmulo de assunto quando voltarem as aulas presenciais. & $302(73,3)$ & $156(63,9)$ & $<0,001$ \\
\hline Não, não estou preocupado com isso. & $25(6,1)$ & $26(10,7)$ & \\
\hline Não penso nisso. & $11(2,7)$ & $24(9,8)$ & \\
\hline A faculdade não aderiu ao EAD. & $74(18)$ & $38(15,6)$ & \\
\hline \multicolumn{4}{|l|}{ Você está com dificuldade de estudar após o isolamento social? } \\
\hline Sim, mas não consigo me concentrar. & $314(76,2)$ & $124(50,8)$ & $<0,001$ \\
\hline Sim, não consigo me adaptar ao EAD. & $51(12,4)$ & $37(15,2)$ & \\
\hline Não, consigo estudar normalmente. & $47(11,4)$ & $83(34)$ & \\
\hline \multicolumn{4}{|l|}{ Você se preocupa com a perda ou atraso do semestre? } \\
\hline Sim, me preocupo bastante com isso. & $327(79,4)$ & $147(60,2)$ & $<0,001$ \\
\hline Não, não estou preocupado. & $61(14,8)$ & $60(24,6)$ & \\
\hline Não penso nisso. & $24(5,8)$ & $37(15,2)$ & \\
\hline
\end{tabular}


Tabela 3. Relação entre a saúde mental da população estudada e a presença de sofrimento psíquico

\begin{tabular}{|c|c|c|c|}
\hline & \multicolumn{2}{|c|}{ SRQ-20 } & \multirow[b]{2}{*}{ Valor-p } \\
\hline & $\begin{array}{c}>7 \\
\mathrm{n}(\%)\end{array}$ & $\begin{array}{c}\leq 7 \\
\text { n (\%) }\end{array}$ & \\
\hline \multicolumn{4}{|l|}{ SAÚDE MENTAL DOS ESTUDANTES DE MEDICINA } \\
\hline \multicolumn{4}{|l|}{ Diagnóstico de transtorno mental prévio } \\
\hline Sim & $157(38,1)$ & $32(13,1)$ & $<0,001$ \\
\hline Não & $255(61,9)$ & $212(86,9)$ & \\
\hline Transtorno de ansiedade generalizada & $112(27,2)$ & $17(7)$ & $<0,001$ \\
\hline Transtornos depressivos & $64(15,5)$ & $13(5,3)$ & $<0,001$ \\
\hline Transtorno bipolar & $7(1,7)$ & $0(0)$ & 0,041 \\
\hline Transtorno da personalidade borderline & $10(2,4)$ & $0(0)$ & 0,014 \\
\hline Transtorno obsessivo-compulsivo & $8(1,9)$ & $3(1,2)$ & 0,492 \\
\hline Transtorno de déficit de atenção e de hiperatividade & $12(2,9)$ & $4(1,6)$ & 0,307 \\
\hline Transtorno do estresse pós-traumático & $2(0,5)$ & $0(0)$ & 0,276 \\
\hline Transtorno explosivo intermitente & $1(0,2)$ & $0(0)$ & 0,441 \\
\hline Transtorno do espectro autista & $1(0,2)$ & $0(0)$ & 0,441 \\
\hline Síndrome de burnout & $1(0,2)$ & $0(0)$ & 0,441 \\
\hline Transtorno de ansiedade social & $1(0,2)$ & $0(0)$ & 0,441 \\
\hline \multicolumn{4}{|c|}{ Deseja ter algum retorno para possível acompanhamento psicoterápico? } \\
\hline $\operatorname{Sim}$ & $116(28,2)$ & $29(11,9)$ & $<0,001$ \\
\hline Não & $296(71,8)$ & $215(88,1)$ & \\
\hline \multicolumn{4}{|c|}{ Você percebeu alguma mudança psicológica ou comportamental de si mesmo durante esses dias? } \\
\hline Sim & $383(93)$ & $151(61,9)$ & $<0,001$ \\
\hline Não & $16(3,9)$ & $59(24,2)$ & \\
\hline Não reparei nisso & $13(3,2)$ & $34(13,9)$ & \\
\hline
\end{tabular}

\section{Aspectos sociais e hábitos de vida durante a pandemia da COVID-19}

Durante a pandemia, 29,3\% dos estudantes não estão em suas cidades de residência, sendo esse um fator impactante na saúde mental. Além disso, 53,7\% dos participantes estão convivendo com alguém que precisa trabalhar fora de casa e demonstram preocupação com isso. Ambos os fatores sugerem correlação com o desenvolvimento de adoecimento mental ( $p=0,001$ e $p<0,001$, respectivamente), como demonstrado na Tabela 4.

Outrossim, a maior parte dos estudantes $(95,4 \%)$ está acompanhada apenas de familiares e do parceiro afetivo, enquanto o restante $(4,6 \%)$ está sozinho ou acompanhado de amigos. A associação entre solidão e adoecimento mental não se mostrou uma evidência significativa $(p=0,365)$.

Apenas 22,2\% relataram conseguir manter uma rotina saudável, como ter uma boa noite de sono, estudar ou realizar alguma atividade física regularmente. A incapacidade de manter hábitos saudáveis durante a pandemia tem impacto negativo na saúde mental dos estudantes $(p<0,001)$.

Foi observado também que 34,4\% tiveram algum conhecido próximo diagnosticado com a COVID-19, 2\% dos participantes já tiveram a infecção pelo novo coronavírus e 3,7\% relataram que alguém do convívio familiar foi infectado previamente. Ademais, 59,3\% dos estudantes de Medicina do Brasil admitem estar com medo de ser infectados, havendo impacto no adoecimento mental $(p<0,001)$.

\section{DISCUSSÃO}

\section{Características sociodemográficas}

No que diz respeito à análise das características da nossa amostra, a maior prevalência de sofrimento psíquico em sujeitos do sexo feminino $(80,6 \%)$ é semelhante aos resultados indicados em estudos prévios. Na literatura, o sexo feminino tem sido descrito como um fator de maior predisposição ao estresse e aos transtornos mentais. Isso tem sido associado aos múltiplos papéis exercidos pela mulher na sociedade juntamente com as alterações hormonais, que interferem no humor dela ${ }^{20-22}$.

Em nossa amostra, também houve maior prevalência na faixa etária entre 18 e 23 anos (70,7\%). Um estudo epidemiológico prévio dos transtornos mentais mostra que adultos jovens, com idade entre 18 e 35 anos, apresentam o principal grupo diagnóstico dos transtornos de humor, representando 
Tabela 4. Relação entre aspectos sociais, hábitos de vida durante a pandemia da COVID-19 e a presença de sofrimento psíquico

\begin{tabular}{|c|c|c|c|}
\hline & \multicolumn{2}{|c|}{ SRQ-20 } & \multirow[b]{2}{*}{ Valor-p } \\
\hline & $\begin{array}{l}>7 \\
\mathrm{n}(\%)\end{array}$ & $\begin{array}{c}\leq 7 \\
\mathrm{n}(\%)\end{array}$ & \\
\hline \multicolumn{4}{|l|}{ A cidade na qual você está durante a pandemia é a sua cidade de residência? } \\
\hline $\operatorname{Sim}$ & $272(66)$ & $192(78,7)$ & 0,001 \\
\hline Não & $140(34)$ & $52(21,3)$ & \\
\hline \multicolumn{4}{|l|}{ Você ou alguém que está na mesma casa que você está trabalhando fora de casa? } \\
\hline Sim, mas não me preocupo com isso. & $35(8,5)$ & $46(18,9)$ & $<0,001$ \\
\hline Sim, e estou preocupado com isso. & $247(60)$ & $105(43)$ & \\
\hline Não. & $130(31,6)$ & $93(38,1)$ & \\
\hline \multicolumn{4}{|l|}{ Você está em casa acompanhado de alguém? } \\
\hline Sim, apenas de familiares. & $328(79,6)$ & $182(74,6)$ & 0,365 \\
\hline Sim, apenas de meu parceiro afetivo. & $11(2,7)$ & $12(4,9)$ & \\
\hline Sim, apenas de amigos. & $7(1,7)$ & $4(1,6)$ & \\
\hline Sim, de familiares e de meu parceiro. & $53(12,9)$ & $40(16,4)$ & \\
\hline Não, estou sozinho. & $13(3,2)$ & $6(2,5)$ & \\
\hline \multicolumn{4}{|l|}{$\begin{array}{l}\text { Você está conseguindo manter uma rotina saudável, como ter um boa noite de sono, estudar } \\
\text { e realizar alguma atividade física regularmente? }\end{array}$} \\
\hline Sim, estou conseguindo manter-me dentro de rotina saudável. & $51(12,4)$ & $95(38,9)$ & $<0,001$ \\
\hline Tenho um boa noite de sono e estudo, mas não faço atividade física. & $55(13,3)$ & $53(21,7)$ & \\
\hline Realizo as atividades diárias, mas não faço atividade física e não tenho um boa noite de sono. & $65(15,8)$ & $22(9)$ & \\
\hline Realizo atividade física e tenho sono regular, porém não consigo estudar. & $54(13,1)$ & $44(18)$ & \\
\hline Não, não consigo manter uma rotina saudável. & $187(45,4)$ & $30(12,3)$ & \\
\hline \multicolumn{4}{|l|}{ Alguém próximo a você foi diagnosticado com a COVID-19? } \\
\hline Sim & $154(37,4)$ & $71(29,1)$ & 0,031 \\
\hline Não & $258(62,6)$ & $173(70,9)$ & \\
\hline \multicolumn{4}{|l|}{ Você ou alguém que está na mesma casa que você foi diagnosticado com a COVID-19? } \\
\hline Sim, eu fui. & $4(1)$ & $9(3,7)$ & 0,041 \\
\hline Sim, alguém que está na mesma casa que eu foi. & $17(4,1)$ & $7(2,9)$ & \\
\hline Não. & $391(94,9)$ & $228(93,4)$ & \\
\hline \multicolumn{4}{|l|}{ Você está com medo de pegar a COVID-19 } \\
\hline Sim & $267(64,8)$ & $122(50)$ & $<0,001$ \\
\hline Não & $60(14,6)$ & $60(24,6)$ & \\
\hline Já tive, porém agora não mais. & $38(9,2)$ & $20(8,2)$ & \\
\hline Não penso nisso. & $47(11,4)$ & $42(17,2)$ & \\
\hline \multicolumn{4}{|l|}{$\begin{array}{l}\text { Durante a pandemia, você está tomando os devidos cuidados (lavagem de mãos constante, } \\
\text { aderindo ao uso de máscaras e do álcool em gel)? }\end{array}$} \\
\hline Totalmente & $369(89,6)$ & $224(91,8)$ & 0,325 \\
\hline Parcialmente & $40(9,7)$ & $20(8,2)$ & \\
\hline Não & $3(0,7)$ & $0(0)$ & \\
\hline
\end{tabular}

$72,8 \%$ dos casos $^{23}$. Isso ocorre porque o jovem entra na, culturalmente, considerada idade ou fase adulta, perpassando por intensas transformações, como o desenvolvimento físico e emocional, em que o indivíduo adota comportamentos influenciados pelo meio socioambiental24.

A literatura também mostra que os distúrbios mentais são mais comuns entre os solteiros do que entre os casados, como em nossa amostra, em que se encontrou a prevalência de sofrimento psíquico nesse grupo (53,5\%), pelo fato de se levar em conta que o casamento poderá proporcionar, além de satisfação sexual, segurança, e que não ser casado ou separar-se seria perturbador para a saúde mental do indivíduo ${ }^{25}$.

Concernente ao ano de graduação, os estudantes com maior sofrimento psíquico se situam nos três primeiros anos de curso. É irrefutável que a correlação do estresse psíquico com as etapas cursadas pelos alunos é crucial na análise do 
desgaste mental. Na análise de Fiorotti, foi observado que a prevalência de transtornos mentais também é maior em discentes do curso básico (43,6\%), em relação aos 40,3\% e 27\% dos alunos do ciclo clínico e do internato, respectivamente ${ }^{26}$.

Analogamente, estudos demonstram que $25,6 \%$ dos alunos referiram quadro depressivo no primeiro ano na Universidade Estadual do Ceará (UECE). Já na Universidade Federal da Bahia (UFBA), o segundo ano estava relacionado com uma maior baixa qualidade de vida, ratificando o predomínio do adoecimento nos ciclos básicos e clínicos ${ }^{27,28}$.

Ademais, outro estudo também evidenciou o pico no terceiro ano de curso em relação aos sintomas depressivos, havendo um aumento gradual de 3,3\% na frequência de ansiedade alta do primeiro para o segundo ano. Uma explicação plausível para tamanha fragilidade durante tais ciclos do curso é a associação entre o contato com fontes geradoras de estresse (dentre as quais se destaca o excesso de informações), o afastamento da parte teórica com a prática e o pouco tempo para conciliar o estudo com o lazer ${ }^{20,26}$.

\section{Indícios de sofrimento psíquico}

Este estudo sinaliza uma possível relação entre o isolamento social enfrentado em razão da pandemia da COVID-19 e os altos níveis de sofrimento psíquico em estudantes de Medicina do Brasil. Nosso estudo demonstrou que $62,8 \%$ dos respondentes sinalizaram sinais de adoecimento mental e é possível correlacionar isso com os sentimentos de solidão, ansiedade e apatia sentidos durante o contexto de pandemia, uma vez que são consequências em tal cenário de isolamento social29. Gradativamente, é capaz de surgir sentimentos crescentes de depressão e estresse, especialmente durante um período de incerteza, podendo haver sérios impactos na saúde pública, aumentando a vulnerabilidade das pessoas à saúde precária e o enfraquecimento da sociedade como um todo 5 .

\section{Atividades acadêmicas e a pandemia da COVID-19}

A maioria dos estudantes está realizando as atividades acadêmicas por meio do $\operatorname{EAD}(76,7 \%)$ e, entre eles, $66,8 \%$ relatam não conseguir ter concentração nos estudos e 39,2\% relatam não conseguir aprender dessa forma. Foi visto que a maior parte se preocupa com relação às reposições futuras das matérias do curso $(69,8 \%)$ e com a perda ou o atraso semestral (72,3\%).

É perceptível que a má adaptação ao EAD, a dificuldade de concentração e a preocupação com o acúmulo de assuntos para o retorno presencial e com a perda ou atraso do semestre demonstraram relação com o adoecimento mental $(p<0,001)$. Relativamente a isso, sabe-se que a alta prevalência do adoecimento ao longo da graduação ocorre pelo sofrimento psíquico crônico e persistente, que causa efeitos danosos e irreversíveis à saúde posteriormente ${ }^{26}$.

\section{Saúde mental dos estudantes de Medicina}

A pesquisa revela que a maioria alega ter notado uma mudança psicológica ou comportamental durante a quarentena, mesmo aqueles indivíduos que afirmam não ter sido diagnosticados com algum transtorno mental prévio (93\% em SQR > 7 e 61,9\% em SQR < 7, resultando em 81,4\% dos indivíduos entrevistados). Não obstante, percebe-se uma associação entre os indivíduos com diagnóstico prévio de transtorno mental e o adoecimento mental durante a pandemia $(p<0,001)$.

Além disso, durante a coleta dos dados, foi apresentada a opção de solicitar retorno para acompanhamento psicológi$\mathrm{co}$, e $22,7 \%$ dos estudantes demonstraram interesse.

\section{Aspectos sociais e hábitos de vida durante a pandemia da COVID-19}

Foi observada uma aflição dos indivíduos avaliados por medo de contrair o vírus (59,3\%) e, a despeito de a grande maioria não conseguir manter uma rotina saudável $(33,1 \%)$, foi visto que a maioria afirma tomar os devidos cuidados para evitar tal enfermidade $(90,4 \%)$. Em congruência com o atual dado, uma pesquisa feita na Universidade de Glasgow afirmou que o aumento do isolamento social, a solidão, a ansiedade, o estresse e uma crise econômica são uma tempestade perfeita para prejudicar a saúde mental e o bem-estar das pessoas ${ }^{30}$.

Outrossim, 29,3\% dos estudantes não estão em suas cidades de residência durante a pandemia, fator de extrema importância, visto que o apoio emocional, especialmente advindo do âmbito familiar, se caracteriza como um mecanismo de proteção aos fatores estressantes, contribuintes para o desenvolvimento de comorbidades mentais. Isso explica o fato de a maioria dos autores citar que estudantes de municípios distantes e afastados do convívio familiar apresentam maior risco de desenvolver transtornos psíquicos, pois aqueles que visitavam menos do que mensalmente os familiares pertencem ao grupo cuja prevalência foi acima de $50 \%$ em transtornos mentais comuns, nos resultados de algumas análises ${ }^{31-33}$.

Apenas 22,2\% relataram conseguir manter uma rotina saudável, como ter uma boa noite de sono, estudar ou realizar alguma atividade física regularmente. A incapacidade de manter hábitos saudáveis durante a pandemia tem impacto negativo na saúde mental dos estudantes $(p<0,001)^{34,35}$.

\section{Limitações do estudo}

Nosso trabalho tem limitações pela data precoce da coleta de dados (maio e junho de 2020) e pelo fato de nosso instrumento SRQ-20 não ser capaz de fazer diagnósticos, ainda que aponte indícios de sofrimento psíquico. Entretanto, novas pesquisas podem preencher essas lacunas e novos dados podem ser coletados neste momento atual, uma vez que o Brasil é considerado o epicentro da pandemia no mundo. Ademais, é possível notar que houve um viés de seleção, 
uma vez que a maior parte da amostra de estudantes é da região Nordeste (residência dos autores deste trabalho).

\section{CONCLUSÃO}

Os indícios de sofrimento psíquico demonstram-se elevados na população estudada. Além disso, também foi possível concluir que há fatores de proteção para o sofrimento psíquico de estudantes de Medicina durante a pandemia da COVID-19; são eles: ser do sexo masculino, ter mais de 29 anos, estar em união estável ou casado, estar nos três últimos anos da graduação, praticar atividades como passatempo e seguir uma rotina saudável, não ter adoecido e não ter parentes com a doença, não trabalhar ou ter alguém trabalhando fora de casa, não ter histórico de transtorno mental prévio e não cursar a graduação em EAD. Diante dos resultados obtidos, é de extrema importância que haja mais discussões que façam mais abordagens sobre saúde mental, objetivando oferecer suporte psicossocial para os estudantes que estão em sofrimento psíquico. Nossos resultados podem fornecer subsídios para o desenvolvimento de ações preventivas para a presença de sintomas de sofrimento mental na população estudada em contexto de pandemias.

\section{CONTRIBUIÇÕES INDIVIDUAIS}

Larissa de Araújo Correia Teixeira - Concepção e desenho dos estudos; elaboração do artigo; aprovação da versão final a ser publicada.

Ricardo Alves Costa - Concepção e desenho dos estudos; análise e interpretação dos dados; elaboração do artigo; aprovação da versão final a ser publicada.

Roberta Machado Pimentel Rebello de Mattos Concepção e desenho dos estudos; revisão crítica do conteúdo intelectual; aprovação da versão final a ser publicada.

Déborah Pimentel - Concepção e desenho dos estudos; revisão crítica do conteúdo intelectual; aprovação da versão final a ser publicada.

\section{CONFLITO DE INTERESSES}

Todos os autores declaram não haver qualquer conflito de interese, em potencial, neste estudo.

\section{AGRADECIMENTOS}

Agradecemos a contribuição técnica de Ikaro Daniel de Carvalho Barreto, doutor em Biometria e Estatística Aplicada, no que se refere ao gerenciamento dos softwares utilizados e à análise dos dados provenientes da presente pesquisa.

\section{REFERÊNCIAS}

1. Lescure FX, Bouadma L, Nguyen D, Parisey M, Wicky PH, Behillil S, et al. Clinical and virological data of the first cases of COVID-19 in Europe: a case series. Lancet Infect Dis. 2020;20(6):697-706.

2. Sanche S, Lin YT, Xu C, Romero-Severson E, Hengartner N, Ke R. Early Release-High Contagiousness and Rapid Spread of Severe Acute Respiratory Syndrome Coronavirus 2. Emerg Infect Dis. 2020;26(7):1470-7.

3. Rodrigues WP. Coronavírus: um problema de saúde pública? Scire Salutis. 2020;10(2): $18-25$

4. Farias HS. 0 avanço da Covid-19 e 0 isolamento social como estratégia para redução da vulnerabilidade. Espaço e Economia. 2020;17.

5. Zaharieva R. The Danger of Social Isolation During a Pandemic [Internet]. European Public Health Alliance. 2020. Disponível em: https://epha.org/the-dangers-of-social-isolationduring-a-pandemic/. Acesso em: 2 mar. 2021.

6. Ornelas J. Suporte social e doença mental. Anal Psicol. 1996;14:263-8.

7. Rodrigues VB, Madeira M. Suporte social e saúde mental: revisão da literatura. Rev Fac Ciênc Saúde. 2009

8. Oliveira DCD. 0 impacto do suporte social, depressão, ansiedade e impulsividade na adolescência: relação com os comportamentos autolesivos e a ideação suicida [tese de mestrado] [Internet]. Lisboa: Instituto Universitário de Ciências Psicológicas, Sociais e da Vida; 2019. Disponível em: http://repositorio.ispa.pt/bitstream/10400.12/7374/1/20839. pdf. Acesso em: 2 mar. 2021.

9. Oliveira MF, Araujo LMB. Saúde mental do estudante de medicina/Mental health of the medicine student. Braz J Develop. 2019:5(11):23440-52.1.

10. Tatebe FM, Taromaru LK, Amorim MR, Nakashima ST, Canova FB, Bonini LMM. Transtornos emocionais em estudantes de medicina. Rev Iniciação Científica ULBRA [Internet]. 2019;(17). Disponível em: http://www.periodicos.ulbra.br/index.php/ic/article/ view/5074/3655. Acesso em: 2 mar. 2021

11. Fernandes MA, Vieira FER, Avelino FVSD, Santos JDM. Prevalence of anxious and depressive symptoms in college students of a public institution. Rev Bras Enferm. 2018;71:2169-75.

12. Conselho Federal de Medicina. Demografia Médica: Brasil possui médicos ativos com CRM em quantidade suficiente para atender demandas da população [Internet]. Portal Médico - Conselho Federal de Medicina. 2018. Disponível em: https://portal.cfm.org.br/index. php?option $=$ com_content\&view $=$ article\&id $=27983: 2018-11-26-13-05-15 \&$ catid $=3$. Acesso em: 2 mar. 2021

13. Fontelles MJ, Simões MG, Almeida JC, Fontelles RGS. Metodologia da pesquisa: diretrizes para o cálculo do tamanho da amostra. Rev Para Med. 2010.

14. Santos KOB, Araújo TM, Oliveira NF. Estrutura fatorial e consistência interna do Self-Reporting Questionnaire (SRQ-20) em população urbana. Cad Saúde Pública. 2009;25:214-22.

15. Fernandes SRP, Almeida Filho N. Validação do SRQ-20 em amostra de trabalhadores de informática. Re Bras Saúde Ocup. 1997;24:105-12.

16. de Jesus Mari J, Williams P. A validity study of a psychiatric screening questionnaire (SRQ20) in primary care in the city of Sao Paulo. Br J Psychiatry. 1986;148(1):23-6.

17. Palácios M, Jardim S, Ramos A, Silva Filho JF. Validação do Self-Report Questionnaire-20 (SRQ-20) numa população de trabalhadores de um banco estatal no Rio de Janeiro, Brasil. In: Silva Filho JF, Jardim S, orgs. A danação do trabalho: organização do trabalho e sofrimento psíquico. Rio de Janeiro: Te Corá Editora; 1998. p. 225-41.

18. Fontelles MJ, Simões MG, Almeida JC, Fontelles RGS. Metodologia da pesquisa: diretrizes para 0 cálculo do tamanho da amostra. Rev Para Med. 2010;24(2):57-64.

19. Miot HA. Tamanho da amostra em estudos clínicos e experimentais. J Vasc Bras. 2011;10(4):275-8.

20. Lima JKA, Brito APA. Desgaste e sofrimento psíquico em estudantes de medicina: uma revisão sistemática. XVII SEPA [Internet]. 2018;17. Disponível em: http://www.revistas. unifacs.br/index.php/sepa. Acesso em: set. 2020.

21. de Paula JA, Borges AMFS, Bezerra LRA, Parente HV, de Paula RCA, Wajnsztejn R, et al. Prevalence and factors associated with depression in medical students. J Hum Growth Develop. 2014;24(3):274-81. 
22. Fouilloux Morales C, Barragán Pérez V, Ortiz León S, Jaimes Medrano A, Urrutia Aguilar ME, Guevara-Guzmán R. Síntomas depresivos y rendimiento escolar en estudiantes de Medicina. Salud Mental. 2013;36(1):59-65.

23. de Miranda CA, Tarasconi CV, Scortegagna SA. Estudo epidêmico dos transtornos mentais. Aval Psicol. 2008;7(2):249-57.

24. Albuquerque C, Cunha M, Matos C, Capela C, Mendes M, Gomes M, et al. Fatores de risco para a saúde mental infanto-juvenil: conhecimentos dos agentes educativos. Acta Paul Enferm [Internet]. 2020;33. Disponível em: http://www.sciel0.br/scielo.php?script=sci_ arttext\&pid=S0103-21002020000100442\&nrm=iso. Acesso em: 2 mar. 2021.

25. Kolb LC. Psiquiatria clínica. 8a ed. Rio de Janeiro: Interamericana; 1976.

26. Fiorotti KP, Rossoni RR, Borges LH, Miranda AE. Transtornos mentais comuns entre os estudantes do curso de medicina: prevalência e fatores associados. J Bras Psiquiatria. 2010;59(1):17-23.

27. Andrade JBC, Sampaio JJC, Farias LM, Melo LP, Sousa DP, Mendonça ALB, et al. Contexto de formação e sofrimento psíquico de estudantes de medicina. Rev Bras Educ Méd. 2014;38:231-42.

28. Gonçalves SS, Silvany Neto AM. Dimensão psicológica da qualidade de vida de estudantes de medicina. Rev Bras Educ Méd. 2013;37(3):385-95.

29. Ministério da Saúde. Fundação Oswaldo Cruz. Centro de Integração de Dados e Conhecimentos para Saúde. Universidade Federal da Bahia. Epidemia da COVID-19 no Brasil: potencial impacto na saúde mental [Internet]. Relatório de Saúde Mental: 2020. Brasil: MS; 2020. Disponível em: https://covid19br.org/main-site-covida/wp-content/
uploads/2020/05/Relat\%C3\%B3rio-Sa\%C3\%BAde-Mental_final_formatado.pdf. Acesso em: set. 2020.

30. Holmes EA, O'Connor RC, Perry VH, Tracey I, Wessely S, Arseneault L, et al. Multidisciplinary research priorities for the COVID-19 pandemic: a call for action for mental health science. Lancet Psychiatry. 2020;7(6):547-60.

31. Vasconcelos TC, Dias BRT, Andrade LR, Melo GF, Barbosa L, Souza E. Prevalence of anxiety and depression symptoms among medicine students. Rev Bras Educ Médica. 2015;39(1):135-42.

32. Ferreira CMG, Kluthcovsky ACGC, Cordeiro TMG. Prevalência de Transtornos Mentais Comuns e Fatores Associados em Estudantes de Medicina: um Estudo Comparativo. Rev Bras Educ Méd. 2016;40:268-77.

33. Dos Santos LS, Ribeiro IJS, Boery EN, Boery RNSO. Qualidade de vida e transtornos mentais comuns em estudantes de medicina. Cogitare Enferm [Internet]. 2017;22(4). Disponível em: http://revistas.ufpr.br/cogitare/article/view/52126. Acesso em: 2 set. 2020.

34. Roxby P. Coronavirus: "Profound" mental health impact prompts calls for urgent research. [Internet]. BBC News. 2020. Disponível em: https://www.bbc.com/news/ health-52295894. Acesso em: 2 mar. 2021.

35. Balmer N. The urgent need for a mental health research response to COVID-19 [Internet]. MQ: Transforming mental health through research. 2020. Disponivel em: https:/www. mqmentalhealth.org/posts/urgent-mental-health-research-response-coronavirus. Acesso em: set. 2020. 